\title{
Study on Clinico Epidemiological Pattern of Syphilis in Patients Attending STD Clinic
}

\author{
Dr. S. Kalaivani \\ Associate Professor of STD, Institute of Venereology, Madras Medical College, Chennai -600003, Tamil Nadu, India
}

\begin{abstract}
Introduction: Syphilis caused by Treponema pallidum is characterized by episodes of active clinical disease interrupted by periods of latent infection. Aim: To study the clinico epidemiological pattern of syphilis and to estimate its regional burden. Methods and Materials: A study was conducted in 6965 patients from January to June 2015 in STD O.P for prevalence of syphilis. Results: Prevalence of syphilis - $4.09 \%$ was similar in both sexes. $31 \%$ of the males were homosexuals. $79 \%$ females and $57 \%$ males were asymptomatic. 64\% of males gave history of sexual exposure. $33 \%$ of female and 9\% of male patients had false positive VDRL. 3 males had cardiovascular syphilis, one patient with neurosyphilis, one 5 months old baby had early congenital syphilis. Conclusion: Majority of them were asymptomatic. Screening and sex education for the reduction of syphilis burden and transmission of HIV to be done.
\end{abstract}

Keywords: Syphilis, Venereal Disease Research Laboratory (VDRL), Treponema Pallidum Haemagglutination (TPHA), Biological False Positive VDRL (BFP), Men Having Sex With Men (MSM)

\section{Introduction}

Syphilis is a sexually transmitted infection which is characterized by episodes of active clinical disease interrupted by periods of latent infection. It is caused by the spirochete Treponema pallidum.

\section{Aim}

To study the clinico epidemiological pattern of syphilis and to estimate its regional burden.

\section{Methods \& Materials}

A observational study was conducted in 6965 patients who attended the std clinic from January to June 2015 was analysed for prevalence of syphilis. Screening of all patients was done with VDRL and subsequent VDRL positive cases were subjected for specific TPHA testing.

\section{Results}

VDRL positivity was $57.4 \%$ in 25 -45years age group, followed by $22 \%, 11.2 \%$ and $9.4 \%$ in the age group of $45-60$ years, $>60$ years and $<25$ years respectively.

Highest level of promiscuity (33\%) in 25-45 years age , followed by $29 \%$, $8 \%$ in the age group of $45-60$ years and $<25$ years respectively.

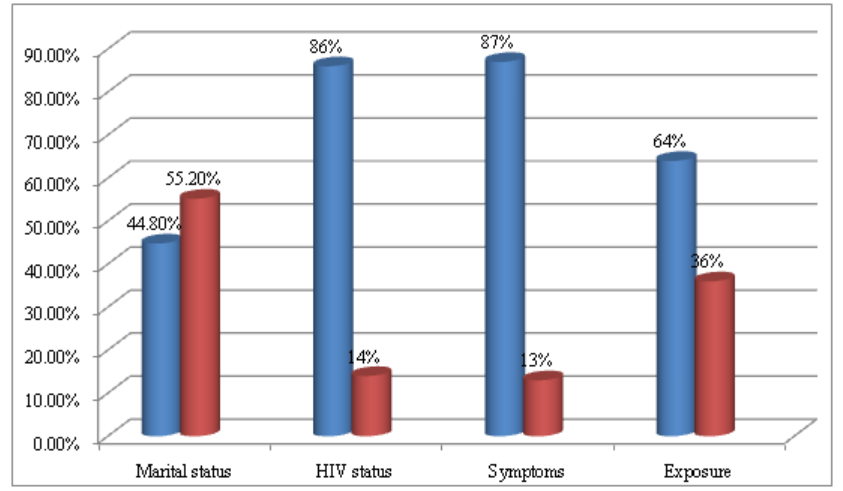

Marital status- Blue: Unmarried

HIV Status- Blue: Negative

Symptoms- Blue: Asymptomatic

H/O Exposure - Blue: Positive

Prevalence of syphilis - $4.09 \%$ (258) which was slightly higher among males than females. $31 \%$ of the males were homosexuals. $83 \%$ of them were referred from other department for routine screening. Only $17 \%$ of them had come for self screening.

Out of the study group $79 \%$ of females and $57 \%$ males were asymptomatic.64\% of males gave the history of sexual exposure and the remaining $36 \%$ denied sexual exposure. $33 \%$ of female and $9 \%$ of male patients had false positive VDRL. $26.7 \%$ of them had primary syphilis.

$44.8 \%$ of the males and $6.25 \%$ of the females were unmarried. 3 males had cardiovascular syphilis, one patient presented with neurosyphilis and one 5 months old baby had early congenital syphilis.

\section{Discussion}

Treponema pallidum is a cork screw shaped microaerophilic bacterium which cannot be cultured in laboratory ${ }^{[1]}$ Because of the inability to grow in culture the diagnosis depends on the history of sexual exposure, recognition of protean clinical signs and symptoms and interpretation of diagnostic tests.

In our observational study all patients attending STD clinic from Jan 15 to June 15 were included for serological analysis. Serological survey continues to be the best source of information on the prevalence of syphilis. All patients tested with both VDRL and specific TPHA $^{\text {[2] }}$

Base line prevalence of syphilis was $4.09 \%$. It is slightly higher in males than females. Prevalence among males$3.99 \%$ and females-3.25\%. This is similar to the study conducted in tertiary care of Northern India ${ }^{[3]} 57 \%$ of the males and $79 \%$ of the females were asymptomatic. 


\section{International Journal of Science and Research (IJSR) \\ ISSN (Online): 2319-7064}

Index Copernicus Value (2013): 6.14 | Impact Factor (2014): 5.611

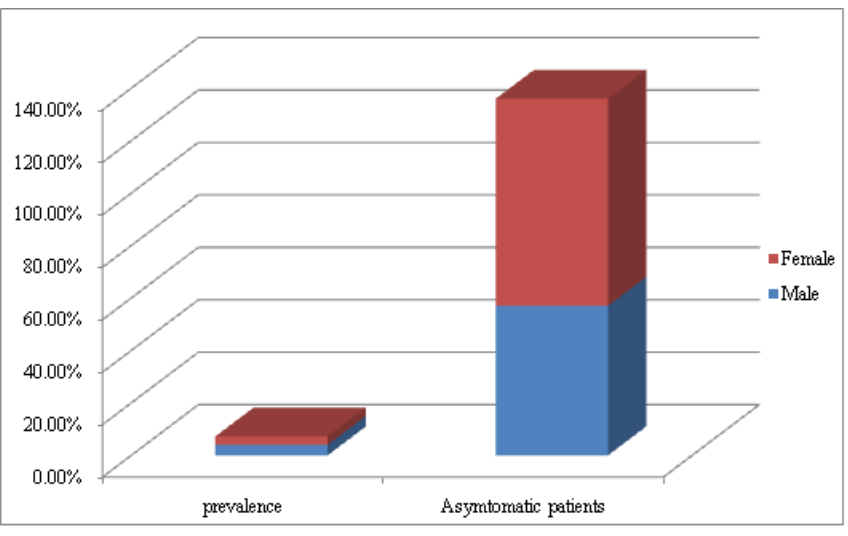

In our study $31 \%$ of the males were Men Having Sex with Men (MSM). The largest increase in the number of syphilis cases has occurred among MSM $^{[4]}$ The increased incidence of syphilis in this populations due in part to rising rates of risky sexual behaviours such as anonymous sex, unprotected sex(oral and anal) and sex with multiple partners. The probability of getting infection through homosexual route is around $30 \%$.

$44.8 \%$ of the males were unmarried. The frequency of syphilis is higher among the single. Broken homes, emotional immaturity and changing behavioral pattern among the adolescents may be the contributing factors. $64 \%$ of the males gives the history of extra marital contact. Sexual dyshormony, prostitution, urbanization \&industrialization and staying away from home are the major contributing factors.

$14 \%$ of the syphilitic patients are co-infected with HIV. This is similar to a population based study in Andhra Pradesh, India ${ }^{[5]}$ The interaction of syphilis and HIV infection is complex and the diagnosis of syphilis is more complicated. High titre of VDRL $>64$ was noted in HIV infected patients ${ }^{[6]}$. Syphilis is associated with an increased risk for HIV infection among both homosexual and heterosexual persons. Presumably sexual behaviours that increase the risk for acquiring syphilis also increase the risk for HIV transmission.

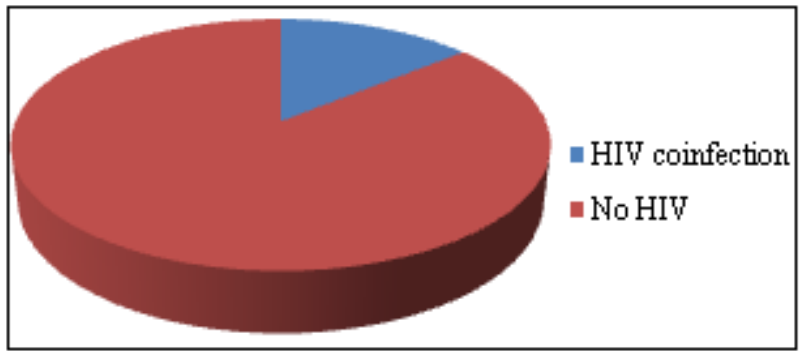

BFP more common women than men (33\% in females and $9 \%$ in males) denotes the presence of non-specific autoantibodies (anti-cardiolipin antibodies) in females. This may be due to the exposure female immune system to fetal antigens during pregnancy.

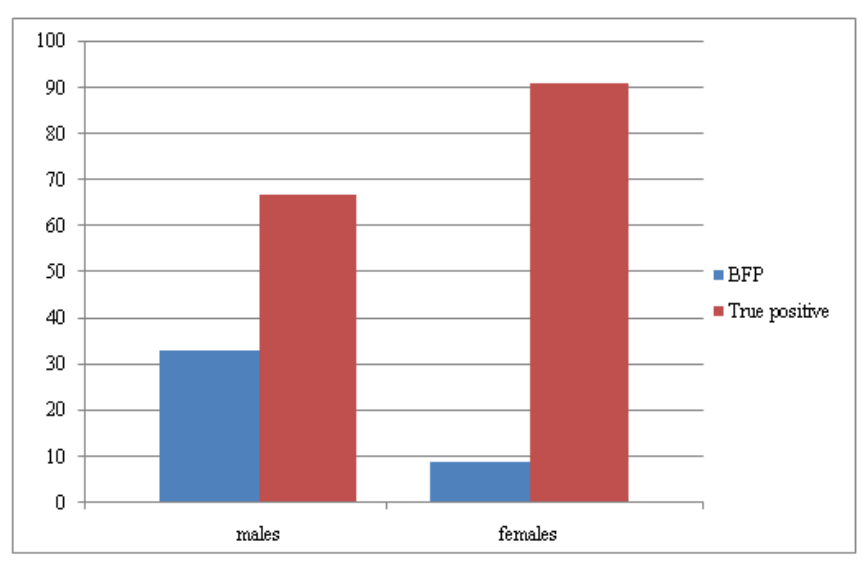

$3.4 \%$ of the patients with HIV co-infection showed BFP VDRL ${ }^{[7]}$ which may be due to antibodies against nonspecific antigens like Reagin, Cardiolipin and Lecithin ${ }^{[8]}$

Asymptomatic patients referred from other department for routine screening revealed more number of positive VDRL and TPHA clearly tells us the burden in the general population. Even though the previous studies shows declining trends of syphilis from 2001 to 2013, the high baseline value of this observational study mandates an intensification of STI control programme.

\section{Conclusion}

All the patients with newly diagnosed syphilis should be counselled for HIV testing and newly diagnosed HIV patients should be screened for syphilis.

Importance of correct and consistent use of condom should be insisted among MSM and others with high risk sexual behaviour. Strengthening of combined HIV-STIs prevention programmes like AVAHAN.

\section{References}

[1] Sexually Transmitted Diseases and HIV/AIDS, Vinod K Sharma ; 2009 : 264

[2] Luger AFH. Serological diagnosis of syphilis: Current methods, In: Young H, McMillian A, eds. Immunological diagnosis of sexually transmitted diseases. New york: Marcel Decker; 1998: p. 249 - 74.

[3] Seroprevalence of syphilis infection among patients attending Antonatal care and Sexually Transmitted Disease (STD) clinics, Observation from tertiary care Hospital of Northern India. Ref : American Journal of Internal Medicine 2014; 2(1) 6 - 9

[4] Kahn RH, Heffelfinger JD, Berman SM. Syphilis outbreaks among men who have sex with men. A public health trend of concern. Sex Transm Dis 2002 ; 29: 285-7

[5] John A. Schneider, Vemu Laxmi et al, Population based seroprevelance of HSV-2 \& Syphilis In Andhra Pradesh., State of India. Ref. BMC Inf. Dis 2010.

[6] Musher DM. Syphilis, neurosyphilis, pencillin and AIDS Ref. J. Infect Dis1991; 163:1201-6.

[7] Association of Biological false positive reactions for Syphilis with HIV Infection : Journal of Infectious Diseases Vol. 165; Issue - 6 p. 1124 -26

[8] Goh BT. Syphilis in adults. Sex Transm Infect 2005; 81: 448-52 